\title{
Clofilium inhibits Slick and Slack potassium channels
}

\author{
This article was published in the following Dove Press journal: \\ Biologics:Targets and Therapy \\ 12 December 2012 \\ Number of times this article has been viewed
}

\author{
Maria de los Angeles Tejada' \\ Kathleen Stolpe' \\ Anne-Kristine Meinild ${ }^{2}$ \\ Dan A Klaerke' \\ 'Department of Physiology and \\ Biochemistry, Faculty of Life Sciences, \\ ${ }^{2}$ Institute of Biology, Faculty of \\ Science, University of Copenhagen, \\ Copenhagen, Denmark
}

Correspondence: Dan A Klaerke Department of Physiology and Biochemistry, Faculty of Life Sciences, University of Copenhagen, Groennegaardsvej 7, 1870 Frederiksberg C, Denmark

Tel +453533 25I I

Fax +4535332525

Emaildk@sund.ku.dk

\begin{abstract}
Slick and Slack high-conductance potassium channels have been recently discovered, and are found in the central nervous system and in the heart. Both channels are activated by $\mathrm{Na}^{+}$ and $\mathrm{Cl}^{-}$, and Slick channels are also inhibited by adenosine triphospate (ATP). An important role of setting the resting membrane potential and controlling the basal excitability of neurons has been suggested for these channels. In addition, no specific blockers for these channels are known up to the present. With the purpose of studying the pharmacological characteristics of Slick and Slack channels, the effects of exposure to the antiarrhythmic compound clofilium were evaluated. Clofilium was able to modulate the activity of Slick and Slack channels effectively, with a stronger effect on Slack than Slick channels. In order to evaluate the pharmacological behavior of Slick and Slack channels further, 38 commonly used potassium channel blockers were tested. Screening of these compounds did not reveal any modulators of Slick and Slack channels, except for clofilium. The present study provides a first approach towards elucidating the pharmacological characteristics of Slick and Slack channels and could be the basis for future studies aimed at developing potent and specific blockers and activators for these channels.
\end{abstract}

Keywords: Slo2.1, Slo2.2, clofilium, potassium channel blockers

\section{Introduction}

Potassium channels are essential for numerous physiological processes, and are involved in a number of disorders, including cardiac arrhythmias, neuronal diseases (episodic ataxia, neurodegeneration), and renal disease (Bartter's syndrome). ${ }^{1}$ A number of small molecules with the ability either to block or to open specific types of potassium channels have been identified, and may be potential drugs for treatment of a number of diseases. ${ }^{2}$ In addition, a number of peptides derived from venom of the scorpion (see below), snakes (dendrotoxin), spiders (hanatoxin, SGTx), snails (conotoxins), and the honey bee (apamin, tertiapin) have been identified as being highly selective blockers of a number of ion channels. Scorpion toxins with the ability to block potassium channels include: charybdotoxin and iberiotoxin, affecting Slo1 channels; margatoxin and agitoxin, modulating Kv1.3 channels; and scyllatoxin and tamapin, affecting Slo1 channels. ${ }^{3}$ In general, peptide toxins (about 20-60 amino acids in length) have high selectivity and bind to relatively large external motifs of potassium channels in contrast with small molecules (molecular weight 200-500), which normally occlude the inner pore. ${ }^{2}$ However, due to differential expression of the numerous potassium channels in distinct cell types and even in specific regions inside cells, development of modulators constitutes a challenge for both academia and pharmaceutical companies. 
Slick (Slo2.1) and Slack (Slo2.2) are potassium channels encoded by the Slo gene family of high-conductance potassium channels. In contrast with Slo1 channels, Slick and Slack channels are not activated by $\mathrm{Ca}^{2+}$, but by $\mathrm{Na}^{+}$ and $\mathrm{Cl}^{-} \cdot{ }^{4}$ Furthermore, Slick channels are also inhibited by adenosine triphosphate (ATP). ${ }^{5}$ Both channels have been found to be widely distributed in the central nervous system and apparently in cardiac myocytes. ${ }^{6,7}$ Given these characteristics, together with their low voltage sensitivity, Slick and Slack channels most likely play an important role in tuning of the resting potential and basal excitability in neurons. The importance of channels setting the resting membrane potential seems to increase during episodes of hypoxia, when the intracellular $\mathrm{Na}^{+}$concentration rises due to failure of $\mathrm{Na}^{+} / \mathrm{K}^{+}-\mathrm{ATP}_{\text {ase }}$. The protective role these channels may have during hypoxia is supported by the fact that mutations of the Slack ortholog in Caenorhabditis elegans result in animals that are hypersensitive to hypoxia. ${ }^{4}$ In addition, it has been shown that Slack channels are activated by estradiol, a fact that could account for estradiol-dependent neuroprotection in ischemia. ${ }^{8}$ Together, these observations raise the possibility that Slick and Slack channels could be used therapeutically as drug targets. Additionally, identification of pharmacological agents targeting these channels will result in important tools for the study of the physiological significance of Slick and Slack channels in different tissues and organs. However, at present, knowledge concerning the pharmacology of Slick and Slack channels is limited, and no specific pharmacological agents targeting Slick and Slack potassium channels have been identified. For Slack channels, antiarrhythmic drugs like quinidine and bepridil have been found to act as inhibitors, and bithionol, an antiparasitic compound, is an activator of Slack channels as well as Slo1 channels. ${ }^{9}$ In addition, it was recently found that niflumic acid and loxapine, an antipsychotic drug, activate Slick and Slack channels, respectively. ${ }^{10,11}$ The present work constitutes a first approach towards analyzing the effect of clofilium, a potent antiarrhythmic compound, on Slick and Slack channels. A number of other well known ion channel modulators were also screened for a potential effect.

\section{Materials and methods}

\section{Heterologous expression of Slick and Slack channels in Xenopus laevis oocytes}

Preparation of Xenopus laevis oocytes was performed as previously described by Grunnet et al. ${ }^{12}$ hSlick and rSlack cDNA cloned into a pOX vector (kindly provided by L Salkoff) was linearized with NotI (New England Biolabs,
Ipswich, MA). mRNA was obtained by in vitro transcription using a mMessage mMachine kit (Ambion, Carlsbad, CA) according to the manufacturer's instructions. RNA was extracted using a MEGAclear kit (Ambion). Next, $50 \mathrm{~nL}$ containing approximately $10 \mathrm{ng}$ of Slick or Slack mRNA was injected into the oocytes, which were cultured in Kulori medium $(90 \mathrm{nM} \mathrm{NaCl}, 1 \mathrm{mM} \mathrm{KCl}, 1 \mathrm{mM} \mathrm{MgCl}, 1 \mathrm{mM}$ $\mathrm{CaCl}_{2}, 5 \mathrm{mM}$ HEPES, $\mathrm{pH} 7.4$ ) at $19^{\circ} \mathrm{C}$ for $4-5$ days prior to our experiments.

\section{Electrophysiological measurements and screening for Slick and Slack blockers}

Expression of Slick and Slack channels was verified by measuring current flow through the channels using a twoelectrode voltage clamp amplifier (Warner Instruments, Novarto, CA). Oocytes were impaled with electrodes pulled from a micropipette puller (P-97, Sutter Instruments, Novato, CA) and filled with $1 \mathrm{M} \mathrm{KCl}$ (resistance about $0.5-1.5 \mathrm{~m} \Omega$ ). Currents were measured either by a stepwise protocol, ie, $500 \mathrm{msec}$ depolarizations from -100 to $+80 \mathrm{mV}$ (holding potential $-80 \mathrm{mV}$ for 4 seconds) or by a pulse protocol, ie, $500 \mathrm{msec}$ depolarizations from -80 to $+80 \mathrm{mV}$ (holding potential $-80 \mathrm{mV}$ for 3 seconds).

In order to screen for compounds able to modulate the activity of Slick and Slack channels, 38 potassium channel blockers (Alomone, Jerusalem, Israel) were dissolved in bath solution (Kulori) immediately before the experiments were conducted. Currents were measured before and after treatment. The compounds tested were E-4031 $(10 \mu \mathrm{M})$, recombinant margatoxin $(300 \mathrm{nM})$, mast cell degranulating peptide $(10 \mu \mathrm{M}), \delta$-dendrotoxin $(1 \mu \mathrm{M})$, dendrotoxin $\mathrm{K}(1 \mu \mathrm{M})$, dendrotoxin-I $(1 \mu \mathrm{M})$, recombinant BeKm-1 (500 nM), recombinant Aa1 $(1.3 \mu \mathrm{M})$, BDS-I $(1.06 \mu \mathrm{M})$, BDS-II $(1.05 \mu \mathrm{M})$, recombinant argitoxin-1 $(500 \mathrm{nM})$, recombinant argitoxin-2 (500 $\mathrm{nM})$, recombinant argitoxin-3 (500 nM), sApamin $(10 \mu \mathrm{M}), \gamma$-dendrotoxin $(1 \mu \mathrm{M}), \alpha$-dendrotoxin $(2 \mu \mathrm{M})$, recombinant charybdotoxin $(500 \mathrm{nM})$, recombinant tityustoxin $\mathrm{K} \alpha(300 \mathrm{nM}), \beta$-dendrotoxin $(2 \mu \mathrm{M})$, recombinant ergotoxin (500 nM), recombinant hongotoxin-1 (955 nM), recombinant OsK (500 $\mathrm{nM})$, recombinant kaliotoxin-1 $(500 \mathrm{nM}), \mathrm{Lq} 2(300 \mathrm{nM})$, recombinant maurotoxin $(600 \mathrm{nM})$, recombinant noxiustoxin $(1 \mu \mathrm{M})$, phrixotoxin-2 $(1 \mu \mathrm{M})$, recombinant scyllatoxin $(500 \mathrm{nM})$, recombinant stichodactyla toxin $(500 \mathrm{nM})$, recombinant stromatoxin $(1.32 \mu \mathrm{M})$, recombinant tertiapin $\mathrm{Q}(1 \mu \mathrm{M})$, recombinant tapamin $(1 \mu \mathrm{M})$, recombinant tertiapin $(1 \mu \mathrm{M})$, recombinant Heteropodatoxin-2 $(1 \mu \mathrm{M})$, recombinant iberiotoxin $(500 \mathrm{nM})$, paxiline $(5 \mu \mathrm{M})$, penitrem $\mathrm{A}(5 \mu \mathrm{M})$, and verruculogen $(5 \mu \mathrm{M})$. 
Paxiline, penitrem A, and verruculogen were dissolved in dimethyl sulfoxide to prepare stocks and then further diluted to final concentrations in Kulori medium. Dimethyl sulfoxide did not exceed $0.01 \%$ in the final solutions used. The solvents used in the present study did not affect currents through Slick or Slack channels. All the blockers were tested at the highest reasonable concentration. The tested concentrations were at least one order of magnitude higher than the effective concentration previously described for each blocker on specific potassium channels.

The effect of the antiarrhythmic compound clofilium was tested at $0.01,0.1,10$, and $100 \mu \mathrm{M}$. Clofilium was directly dissolved in Kulori medium prior to application to oocytes expressing Slick or Slack channels. Currents were measured before and after application of treatment. Current values from endogenous channels of noninjected oocytes were subtracted from currents measured for oocytes expressing Slick and Slack channels. The expression of normalized current as a function of drug concentration allowed fitting the dose-response curves with a Hill equation, using a nonlinear regression analysis on $\mathrm{GraphPad}$ Prism $5^{\circledR}$ (Windows GraphPad software, San Diego, CA). The data are shown as the mean and the standard error. A higher concentration of clofilium was also tested, but was not included in the dose-response curves due to the possibility of nonspecific effects on the targeted channels.

Data acquisition and analysis were performed using the following packages: pClamp ${ }^{\circledR}$ (Molecular Devices, San Jose, CA), GraphPadPrism $5^{\circledR}$, and MicrosoftExcel ${ }^{\circledR}$. Sequences of Slick, Slack, and hERG channels were analyzed using the CLC Main Workbench 6.5 (Cambridge, MA). Sequences were aligned at the pore region and S6 transmembrane segment in Slick, Slack, and hERG channels. Conservation of residues was evidenced by sequence logos.

\section{Results}

\section{Effect of clofilium on Slick and Slack channels}

The effect of clofilium on Slick and Slack potassium channels expressed in $X$. laevis oocytes was analyzed using a two-electrode voltage clamp. Figure 1A and B shows Slick and Slack currents measured before (black) and after (red) application of $100 \mu \mathrm{M}$ clofilium. As evident from the current traces, clofilium was able to reduce the activity of Slack channels with a stronger potency compared with its effect on Slick channels. The blocking effect of clofilium on Slick and Slack channels was readily reversible upon washing with the culture medium (data not shown). In the
A

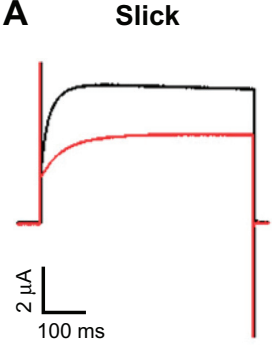

C

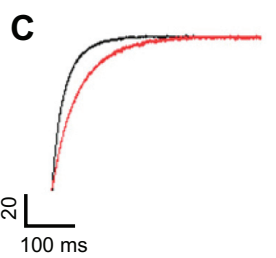

B

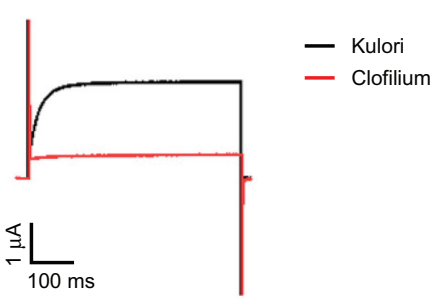

D

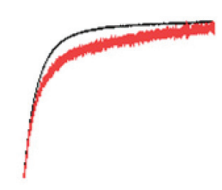

Figure I Effect of clofilium on Slick and Slack potassium channels. Upper panels show current traces of representative Xenopus laevis oocytes expressing either Slick (A) or Slack (B) channels before (black) and after (red) treatment with clofilium $100 \mu \mathrm{M}$. Currents were stimulated by a pulse protocol with $500 \mathrm{msec}$ depolarizations from -80 to $+80 \mathrm{mV}$ from a holding potential of $-80 \mathrm{mV}$ for 3 seconds. Activation kinetics of Slick (C) and Slack (D) in representative oocytes are shown in the lower panels.

Notes: Recorded currents were normalized to maximal current in each experiment. Currents measured in uninjected oocytes were $<0.150 \mu \mathrm{A}$.

experiments shown in Figure 1C and D, we examined the effect of clofilium on channel kinetics, and it can be seen that clofilium slows the activation kinetics of both channels.

To determine the affinity of clofilium, we plotted currents through Slick and Slack channels upon application of increasing concentrations of clofilium (Figure 2A and B). Concentrations of clofilium up to $10 \mu \mathrm{M}$ did not exert any significant blocking effect in either Slick or Slack channels. However, at a concentration of $100 \mu \mathrm{M}$, clofilium was able to inhibit currents through Slick and Slack channels to $75 \% \pm 1 \%$ and $51 \% \pm 3 \%$ of control values, respectively. As a consequence, the effect of this compound is more potent on Slack channels than on Slick channels,indicating that Slack channels are probably significantly more sensitive than Slick channels to clofilium.

\section{Screening of potassium channel blockers for Slick and Slack}

Since knowledge of the pharmacology of Slick and Slack channels is sparse, a set of well known blockers, covering most potassium channels in the genome, was tested to determine their effect on Slick and Slack channels. A total of 38 compounds, including toxins and small peptides, were used for this purpose. Some of the potassium channel blockers tested have been previously shown to have a potent effect on other related potassium channels, eg, iberitoxin and charybdotoxin in Slo1 channels, ${ }^{13,14}$ and apamin and scyllatoxin in SK channels. Furthermore, blockers of Kir 
A

Slick

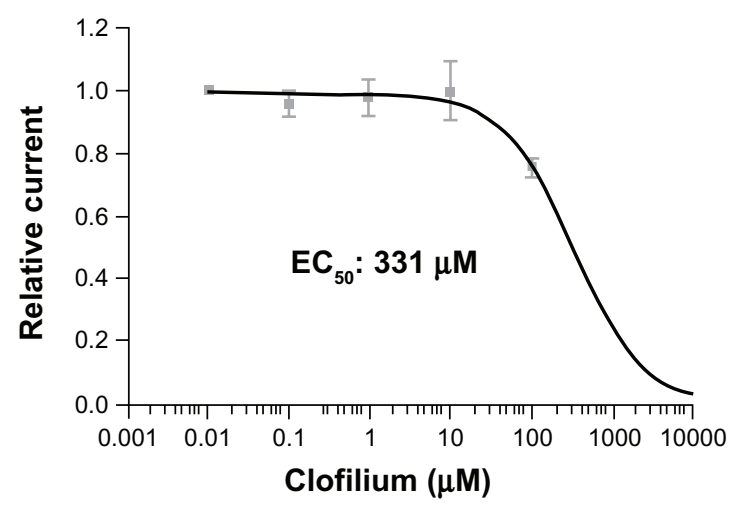

B Slack

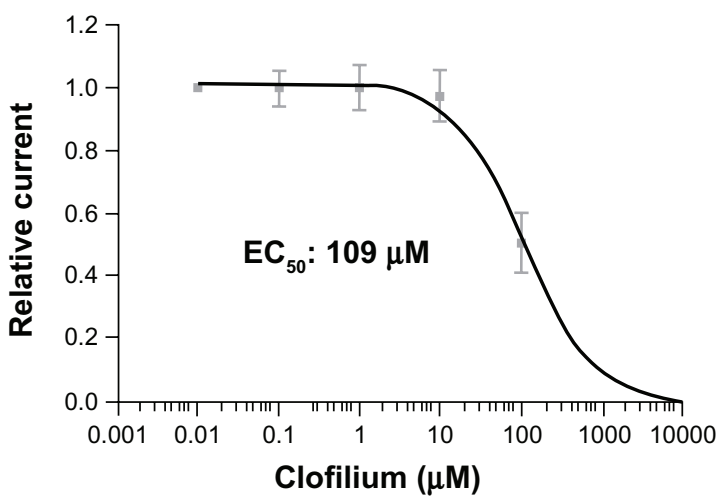

Figure 2 Dose response of Slack and Slick channels upon treatment with clofilium. Oocytes expressing Slick (A) or Slack (B) channels were exposed to different concentrations of clofilium $(0.0 \mathrm{I}-100 \mu \mathrm{M}) . \mathrm{K}^{+}$currents were measured at the end of depolarizing steps of a pulse protocol $(500 \mathrm{msec}$ depolarizations from -80 to $+80 \mathrm{mV}$, holding potential $-80 \mathrm{mV}$

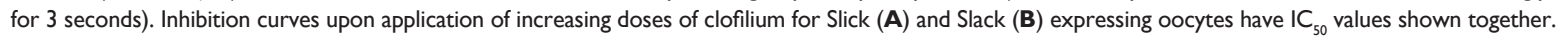

Note: $n=4-8 \pm$ standard error.

channels and $\mathrm{Kv}$ channels, such as tertiapin, agitoxins, dendrotoxins, and margatoxin, which are known to block these channels at nanomolar doses, were also tested. None of the tested compounds was able to significantly modulate the activity of Slick or Slack channels to a significant extent (Figure 3).

\section{Discussion}

The use of channel blockers or openers are important tools in order to be able to understand better the roles Slick and Slack channels may play under physiological and pathophysiological conditions. The present study provides evidence for the effect of clofilium on Slick and Slack potassium channels. We observed that both channels are inhibited after treatment with clofilium, and the effect of this compound seems to be more potent on Slack than Slick channels. Our experiments also show that clofilium affects channel kinetics, resulting in slower activation of both Slick and Slack channels as compared with controls. The slower gating behavior probably underlies the progressive blocking action of clofilium of open channels and a slower onset of channel inhibition, as has been suggested for hERG and hEAG channels. ${ }^{15}$

Clofilium has been shown to block a number of potassium channels with different potency, including hERG $\left(\mathrm{IC}_{50} 2.5 \mathrm{nM}\right)$, $\mathrm{hEAG}\left(\mathrm{IC}_{50} 255 \mathrm{nM}\right),{ }^{15} \mathrm{Kv} 1.5\left(\mathrm{IC}_{50} 840 \mathrm{nM}\right),{ }^{16}$ and

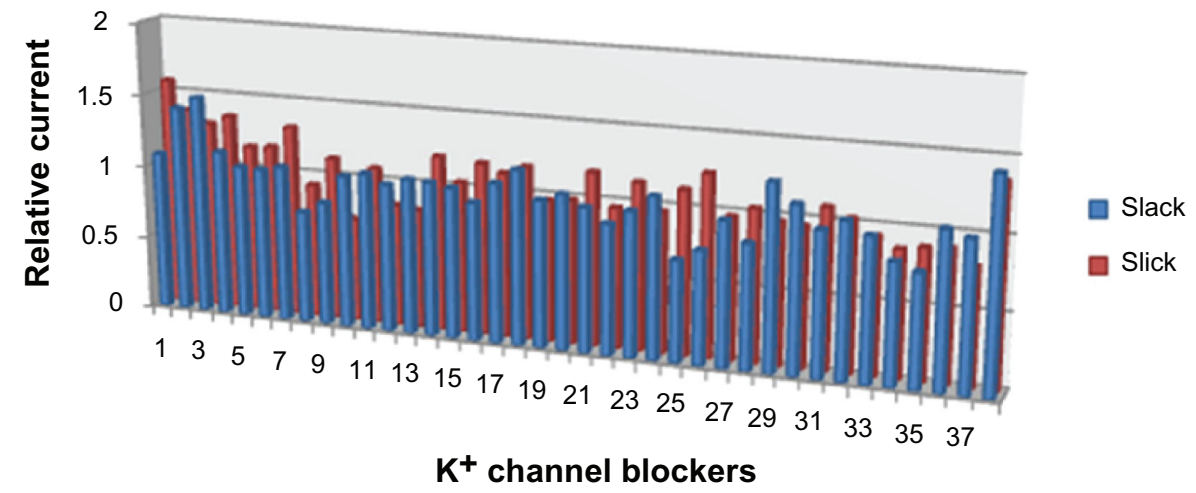

Figure 3 Effect of different $\mathrm{K}^{+}$channel blockers on Slick and Slack channels.

Notes: A set of 38 potassium channel blockers were applied to oocytes expressing Slick and Slack channels. Currents were measured before and after treatment by a stepwise protocol ( -100 to $+80 \mathrm{mV}$, from a holding potential of $-80 \mathrm{mV}$ and depolarizing steps of 500 msec every 3 seconds). Bars represent relative currents at the end of the $+80 \mathrm{mV}$ step. Compounds tested were E-403I (I0 $\mu \mathrm{M})$, recombinant margatoxin $(300 \mathrm{nM})$, mast cell degranulating peptide (I0 $\mu \mathrm{M})$, $\delta$-dendrotoxin (I $\mu \mathrm{M})$, dendrotoxin $\mathrm{K}(\mathrm{I} \mu \mathrm{M})$, dendrotoxin-I (I $\mu \mathrm{M})$, recombinant BeKm-I (500 nM), recombinant Aal (I.3 $\mu \mathrm{M})$, BDS-I (I.06 $\mu \mathrm{M})$, BDS-II (I.05 $\mu \mathrm{M})$, recombinant argitoxin-I $(500 \mathrm{nM})$, recombinant argitoxin-2 (500 nM), recombinant argitoxin-3 (500 nM), sApamin (I0 $\mu M)$, $\gamma$-dendrotoxin $(I \mu M)$, $\alpha$-dendrotoxin $(2 \mu M)$, recombinant charybdotoxin $(500 \mathrm{nM})$, recombinant tityustoxin $\mathrm{K} \alpha(300 \mathrm{nM})$, $\beta$-dendrotoxin $(2 \mu \mathrm{M})$, recombinant ergotoxin $(500 \mathrm{nM})$, recombinant hongotoxin-I (955 nM), recombinant OsK (500 nM), recombinant kaliotoxin-I (500 nM), rLq2 (300 nM), recombinant maurotoxin (600 nM), recombinant noxiustoxin (I $\mu$ M), phrixotoxin-2 (I $\mu \mathrm{M})$, recombinant scyllatoxin $(500 \mathrm{nM})$, recombinant stichodactyla toxin $(500 \mathrm{nM})$, recombinant stromatoxin (I.32 $\mu \mathrm{M})$, recombinant tertiapin $\mathrm{Q}(\mathrm{I} \mu \mathrm{M})$, recombinant tapamin $(I \mu M)$, recombinant tertiapin $(I \mu M)$, recombinant Heteropodatoxin-2 (I $\mu M)$, recombinant iberiotoxin $(500 \mathrm{nM})$, paxiline $(5 \mu \mathrm{M})$, penitrem $\mathrm{A}$ $(5 \mu \mathrm{M})$, and verruculogen $(5 \mu \mathrm{M})$. 


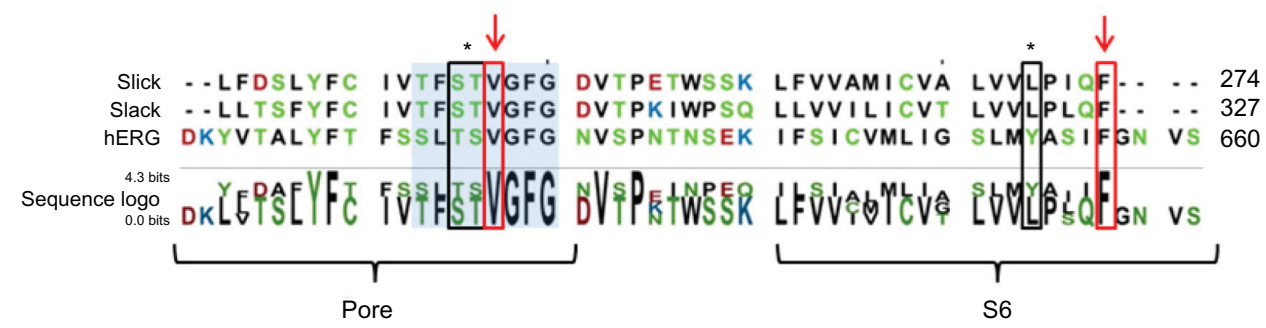

Figure 4 Potential residues involved in the interaction of clofilium with Slick and Slack channels and sequence alignment for the pore helix through the S6 transmembrane segment of Slick, Slack, and hERG channels.

Notes: The blue box indicates the selectivity filter. The red boxes and red arrows indicate two residues (valine and phenylalanine) that have been shown to interact with clofilium in hERG channels and are conserved in Slick and Slack. Black boxes and stars indicate three residues (threonine, serine, and lysine) that have been shown to interact with clofilium in hERG channels but are not conserved in Slick and Slack. The relative positions of the pore helix and S6 are also indicated.

KCNQ1-KCNE1 $\left(\mathrm{IC}_{50}\right.$ about $\left.100 \mu \mathrm{M}\right),{ }^{17,18}$ and recently another member of the high-conductance potassium channel family, ie, the Slo3 channel. ${ }^{19}$ Within the Slo gene family, clofilium blocks Slo3 channels in micromolar concentrations $\left(\mathrm{IC}_{50}\right.$ $50 \mu \mathrm{M}),{ }^{19}$ whereas it does not seem to affect Slo1 channels. ${ }^{20}$ In our hands, clofilium $100 \mu \mathrm{M}$ blocked $50 \%$ of currents through Slack channels, whereas the same concentration blocked Slick channels to $70 \%$ of control levels. Consequently, these members of the Slo2 gene family, despite being highly related in structure and localization, have different sensitivity for this potent antiarrhythmic agent. Given that relatively high doses of clofilium were needed to block these channels, clofilium should not be regarded as a specific blocker of Slick and Slack channels. However, it should be borne in mind that during treatment with this compound, not only hERG and hEAG channels are potently affected, but other channels such as KCNQ1/KCNE1, Slick, and Slack could also be affected. It is noteworthy that clofilium blockade in $X$. laevis oocytes needs doses at least 10 times higher to have a visible effect on hERG channels compared with mammalian cells. ${ }^{15}$ In addition, Gessner and Heinemann ${ }^{15}$ have suggested that the effective concentration of clofilium in cytoplasm is several orders of magnitude lower than that outside the cell. Furthermore, Malayev et $\mathrm{al}^{16}$ have reported that clofilium block on Kv1.5 channels is more than two-fold stronger when applied from the intracellular side. Consequently, it is possible that lower doses of clofilium could exert a stronger effect on Slick and Slack channels expressed in mammalian cell lines and/or when applied as inside-out patches.

It has been shown that certain residues in the pore helix and the S6 transmembrane segment of hERG channels are involved in the interaction with clofilium. ${ }^{21}$ In an attempt to suggest possible interaction sites of clofilium with Slick and Slack channels, we performed a sequence alignment between these channels and hERG. The alignment revealed residues which could be involved in the interaction of clofilium with
Slick and Slack channels. The alignment showed a highly conserved valine at the selectivity filter, together with a residue in the S6 segment, namely phenylalanine, at position F274 for Slick and F327 for Slack, which could probably be involved in the interaction of clofilium with these channels, given that these residues have been shown to bind clofilium in hERG channels (Figure 4). Other residues of hERG channels have also been related to clofilium interaction, ie, threonine T623, serine S624, and tyrosine Y652. However, these residues are not highly conserved between Slick, Slack, and hERG channels (Figure 4), and it could be speculated that this is the reason for the difference in clofilium sensitivity.

In a search for pharmacological agents affecting Slick and Slack channels, a screen of a series of commonly used potassium channel blockers was performed. Among the 38 compounds used, between toxins and small peptides, none of them was able to modulate the activity of Slick or Slack channels to a significant extent. This is the first report that provides evidence from a basic screen of different potassium channel blockers on Slick and Slack channels. Like every first screen, it constitutes a starting point towards unveiling the pharmacological characteristics of these channels. Further research is needed to identify potent and, in the best case, specific blockers of Slick and Slack channels.

\section{Conclusion}

The present study aimed to elucidate the pharmacological characteristics of Slick and Slack high-conductance potassium channels. We were able to identify clofilium as a modulator of the activities of these channels. We suggest that the potent effect of clofilium as an antiarrhythmic could be explained by its action on different potassium channels, in which Slick and Slack may be implicated. We have also presented evidence of other potassium channel blockers not having a significant effect on Slick or Slack channels. It will be interesting in future experiments to tailor small peptides 
according to the structure of the channels to act as specific blockers for either of these channels.

\section{Acknowledgments}

Ms Z Rasmussen is thanked for her expert technical assistance. This work was supported by grants from The Danish Medical Research Council, The Carlsberg Foundation, The Novo Nordic Foundation, The Lundbeck Foundation (Lucens), and The Fougner-Hartmann Foundation.

\section{Disclosure}

The authors declare no conflicts of interest, financial or otherwise, in this work.

\section{References}

1. Shieh CC, Coghlan M, Sullivan JP, et al. Potassium channels: molecular defects, diseases, and therapeutic opportunities. Pharmacol Rev. 2000;52(4):557-594.

2. Wulff H, Castle NA, Pardo LA. Voltage-gated potassium channels as therapeutic targets. Nat Rev Drug Discov. 2009;8(12):982-1001.

3. Jenkinson DH. Potassium channels - multiplicity and challenges. $\mathrm{Br}$ Pharmacol. 2006;147 Suppl 1:S63-S71.

4. Yuan A, Santi CM, Wei A, et al. The sodium-activated potassium channel is encoded by a member of the Slo gene family. Neuron. 2003;37:765-773.

5. Bhattacharjee A, Joiner WJ, Wu M, et al. Slick (Slo2.1), a rapidly-gating sodium-activated potassium channel inhibited by ATP. J Neurosci. 2003;23(37):11681-11691.

6. Bhattacharjee A, Gan L, Kaczmarek LK. Localization of the Slack potassium channel in the rat central nervous system. J Comp Neurol. 2002;454(3):241-254.

7. Bhattacharjee A, von Hehn CA, Mei X, et al. Localization of the $\mathrm{Na}^{+}-$ activated $\mathrm{K}^{+}$channel Slick in the rat central nervous system. J Comp Neurol. 2005;484(1):80-92.

8. Zhang L, Sukhareva M, Barker JL, et al. Direct binding of estradiol enhances Slack (sequence like a calcium-activated potassium channel) channels' activity. Neuroscience. 2005;131(2):275-282.
9. Yang B, Gribkoff VK, Pan J, et al. Pharmacological activation and inhibition of Slack (Slo2.2) channels. Neuropharmacology. 2006;51(4):896-906.

10. Biton B, Sethuramanujam S, Picchione K, et al. The antipsychotic drug loxapine is an opener of the Na+-activated potassium channel Slack (Slo2.2). J Pharmacol Exp Ther. 2011;340 (3):706-715.

11. Dai L, Garg V, Sanguinetti MC. Activation of Slo2.1 channels by niflumic acid. J Gen Physiol. 2010;135(3):275-295.

12. Grunnet M, MacAulay N, Jorgensen NK, et al. Regulation of cloned, $\mathrm{Ca}^{2+}$-activated $\mathrm{K}^{+}$channels by cell volume changes. Pflugers Arch. 2002;444(1-2):167-177.

13. Gimenez-Gallego G, Navia MA, Reuben JP, et al. Purification, sequence, and model structure of charybdotoxin, a potent selective inhibitor of calcium-activated potassium channels. Proc Natl Acad Sci US A. 1988;85(10):3329-3333.

14. Galvez A, Gimenez-Gallego G, Reuben JP, et al. Purification and characterization of a unique, potent, peptidyl probe for the high conductance calcium-activated potassium channel from venom of the scorpion Buthus tamulus. J Biol Chem. 1990;265(19):11083-11090.

15. Gessner G, Heinemann SH. Inhibition of hEAG1 and hERG1 potassium channels by clofilium and its tertiary analogue LY97241. Br J Pharmacol. 2003;138(1):161-171.

16. Malayev AA, Nelson DJ, Philipson LH. Mechanism of clofilium block of the human Kv1.5 delayed rectifier potassium channel. Mol Pharmacol. 1995;47(1):198-205.

17. Honore E, Attali B, Romey G, et al. Cloning, expression, pharmacology and regulation of a delayed rectifier $\mathrm{K}^{+}$channel in mouse heart. EMBOJ. 1991;10(10):2805-2811.

18. Yang WP, Levesque PC, Little WA, et al. KvLQT1, a voltage-gated potassium channel responsible for human cardiac arrhythmias. Proc Natl Acad Sci U S A. 1997;94(8):4017-4021.

19. Zeng XH, Yang C, Kim ST, et al. Deletion of the Slo3 gene abolishes alkalization-activated $\mathrm{K}^{+}$current in mouse spermatozoa. Proc Natl Acad Sci U S A. 2011;108(14):5879-5884.

20. Fernández-Fernández JM, Nobles $\mathrm{M}$, Currid A, et al. Maxi $\mathrm{K}^{+}$ channel mediates regulatory volume decrease response in a human bronchial epithelial cell line. Am J Physiol Cell Physiol. 2002;283(6): C1705-C1714.

21. Perry M, de Groot MJ, Helliwell R, et al. Structural determinants of HERG channel block by clofilium and ibutilide. Mol Pharmacol. 2004;66(2):240-249.
Biologics: Targets \& Therapy

\section{Publish your work in this journal}

Biologics: Targets \& Therapy is an international, peer-reviewed journal focusing on the patho-physiological rationale for and clinical application of Biologic agents in the management of autoimmune diseases, cancers or other pathologies where a molecular target can be identified. This journal is indexed on PubMed Central, CAS, EMBase, Scopus

\section{Dovepress}

and the Elsevier Bibliographic databases. The manuscript management system is completely online and includes a very quick and fair peerreview system, which is all easy to use. Visit http://www.dovepress. com/testimonials.php to read real quotes from published authors. 